\title{
Limit Synchronization in Markov Decision Processes*
}

\author{
Laurent Doyen $^{1}$, Thierry Massart ${ }^{2}$, and Mahsa Shirmohammadi ${ }^{1,2}$ \\ ${ }^{1}$ LSV, ENS Cachan \& CNRS, France \\ 2 Université Libre de Bruxelles, Belgium
}

\begin{abstract}
Markov decision processes (MDP) are finite-state systems with both strategic and probabilistic choices. After fixing a strategy, an MDP produces a sequence of probability distributions over states. The sequence is eventually synchronizing if the probability mass accumulates in a single state, possibly in the limit. Precisely, for $0 \leq p \leq 1$ the sequence is $p$-synchronizing if a probability distribution in the sequence assigns probability at least $p$ to some state, and we distinguish three synchronization modes: $(i)$ sure winning if there exists a strategy that produces a 1-synchronizing sequence; (ii) almost-sure winning if there exists a strategy that produces a sequence that is, for all $\epsilon>0$, a (1- $\epsilon$ )synchronizing sequence; (iii) limit-sure winning if for all $\epsilon>0$, there exists a strategy that produces a (1- $\epsilon)$-synchronizing sequence. We consider the problem of deciding whether an MDP is sure, almost-sure, or limitsure winning, and we establish the decidability and optimal complexity for all modes, as well as the memory requirements for winning strategies. Our main contributions are as follows: (a) for each winning modes we present characterizations that give a PSPACE complexity for the decision problems, and we establish matching PSPACE lower bounds; (b) we show that for sure winning strategies, exponential memory is sufficient and may be necessary, and that in general infinite memory is necessary for almost-sure winning, and unbounded memory is necessary for limitsure winning; (c) along with our results, we establish new complexity results for alternating finite automata over a one-letter alphabet.
\end{abstract}

\section{Introduction}

Markov decision processes (MDP) are finite-state stochastic models used in the design of systems that exhibit both controllable and stochastic behavior, such as in planning, randomized algorithms, and communication protocols [2]13]4. The controllable choices along the execution are fixed by a strategy, and the stochastic choices describe the system response. When a strategy is fixed in an MDP, the symbolic outcome is a sequence of probability distributions over states of the MDP, which differs from the traditional semantics where a probability measure

\footnotetext{
* This work was partially supported by the Belgian Fonds National de la Recherche Scientifique (FNRS), and by the PICS project Quaverif funded by the French Centre National de la Recherche Scientifique (CNRS).
} 
is considered over sets of sequences of states. This view is adequate in many applications, such as systems biology, sensor networks, robot planning, etc. 15 15], where the system consists of several copies of the same process (molecules, sensors, robots, etc.), and the relevant information along the execution of the system is the number of processes in each state, or the relative frequency (i.e., the probability) of each state. In recent works, the verification of quantitative properties of the symbolic outcome was shown undecidable [18. Decidability is obtained for special subclasses [6], or through approximations [1].

In this paper, we consider a general class of strategies that select actions depending on the full history of the system execution. In the context of several identical processes, the same strategy is used in every process, but the internal state of each process need not be the same along the execution, since probabilistic transitions may have different outcome in each process. Therefore, the execution of the system is best described by the sequence of probability distributions over states along the execution. Previously, the special case of word-strategies have been considered, that at each step select the same control action in all states, and thus only depend on the number of execution steps of the system. Several problems for MDPs with word-strategies (also known as probabilistic automata) are undecidable 3141811. In particular the limit-sure reachability problem, which is to decide whether a given state can be reached with probability arbitrarily close to one, is undecidable for probabilistic automata 14.

We establish the decidability and optimal complexity of deciding synchronizing properties for the symbolic outcome of MDPs under general strategies. Synchronizing properties require that the probability distributions tend to accumulate all the probability mass in a single state, or in a set of states. They generalize synchronizing properties of finite automata [20]10]. Formally for $0 \leq p \leq 1$, a sequence $\bar{X}=X_{0} X_{1} \ldots$ of probability distributions $X_{i}: Q \rightarrow[0,1]$ over state space $Q$ of an MDP is eventually p-synchronizing if for some $i \geq 0$, the distribution $X_{i}$ assigns probability at least $p$ to some state. Analogously, it is always $p$-synchronizing if in all distributions $X_{i}$, there is a state with probability at least $p$. For $p=1$, these definitions are the qualitative analogous for sequences of distributions of the traditional reachability and safety conditions [9]. In particular, an eventually 1-synchronizing sequence witnesses that there is a length $\ell$ such that all paths of length $\ell$ in the MDP reach a single state, which is thus reached synchronously no matter the probabilistic choices.

Viewing MDPs as one-player stochastic games, we consider the following traditional winning modes (see also Table 1): $(i)$ sure winning, if there is a strategy that generates an \{eventually, always 1 -synchronizing sequence; (ii) almost-sure winning, if there exists a strategy that generates a sequence that is, for all $\epsilon>0$,

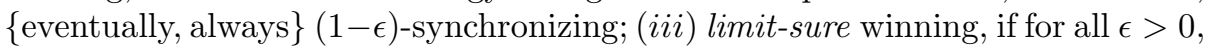
there is a strategy that generates an \{eventually, always $(1-\epsilon)$-synchronizing sequence.

We show that the three winning modes form a strict hierarchy for eventually synchronizing: there are limit-sure winning MDPs that are not almost-sure 
Table 1. Winning modes and synchronizing objectives (where $\mathcal{M}_{n}^{\alpha}(T)$ denotes the probability that under strategy $\alpha$, after $n$ steps the $\operatorname{MDP} \mathcal{M}$ is in a state of $T$ )

\begin{tabular}{|c|c|c|}
\hline & Always & Eventually \\
\hline Sure & $\exists \alpha \quad \forall n \quad \mathcal{M}_{n}^{\alpha}(T)=1$ & $\exists \alpha \quad \exists n \quad \mathcal{M}_{n}^{\alpha}(T)=1$ \\
\hline Almost-sure & $\exists \alpha \quad \inf _{n} \mathcal{M}_{n}^{\alpha}(T)=1$ & $\exists \alpha \sup _{n} \mathcal{M}_{n}^{\alpha}(T)=1$ \\
\hline Limit-sure & $\sup _{\alpha} \inf _{n} \mathcal{M}_{n}^{\alpha}(T)=1$ & $\sup _{\alpha} \sup _{n} \mathcal{M}_{n}^{\alpha}(T)=1$ \\
\hline
\end{tabular}

winning, and there are almost-sure winning MDPs that are not sure winning. For always synchronizing, the three modes coincide.

For each winning mode, we consider the problem of deciding if a given initial distribution is winning. We establish the decidability and optimal complexity bounds for all winning modes. Under general strategies, the decision problems have much lower complexity than with word-strategies. We show that all decision problems are decidable, in polynomial time for always synchronizing, and PSPACE-complete for eventually synchronizing. This is also in contrast with almost-sure winning in the traditional semantics of MDPs, which is solvable in polynomial time for both safety and reachability objectives [8]. Our complexity results are shown in Table 2 .

We complete the picture by providing optimal memory bounds for winning strategies. We show that for sure winning strategies, exponential memory is sufficient and may be necessary, and that in general infinite memory is necessary for almost-sure winning, and unbounded memory is necessary for limit-sure winning.

Some results in this paper rely on insights related to games and alternating automata that are of independent interest. First, the sure-winning problem for eventually synchronizing is equivalent to a two-player game with a synchronized reachability objective, where the goal for the first player is to ensure that a target state is reached after a number of steps that is independent of the strategy of the opponent (and thus this number can be fixed in advance by the first player). This condition is stronger than plain reachability, and while the winner in two-player reachability games can be decided in polynomial time, deciding the winner for synchronized reachability is PSPACE-complete. This result is obtained by turning the synchronized reachability game into a one-letter alternating automaton for which the emptiness problem (i.e., deciding if there exists a word accepted by the automaton) is PSPACE-complete [1617. Second, our PSPACE lower bound for the limit-sure winning problem in eventually synchronizing uses a PSPACEcompleteness result that we establish for the universal finiteness problem, which is to decide, given a one-letter alternating automata, whether from every state the accepted language is finite.

A full version of this paper with all proofs is available [12].

\section{Markov Decision Processes and Synchronization}

A probability distribution over a finite set $S$ is a function $d: S \rightarrow[0,1]$ such that $\sum_{s \in S} d(s)=1$. The support of $d$ is the set $\operatorname{Supp}(d)=\{s \in S \mid d(s)>0\}$. 
We denote by $\mathcal{D}(S)$ the set of all probability distributions over $S$. For $T \neq \emptyset$, the uniform distribution on $T$ assigns probability $\frac{1}{|T|}$ to every state in $T$. Given $s \in S$, the Dirac distribution on $s$ assigns probability 1 to $s$, and by a slight abuse of notation, we usually denote it simply by $s$.

\subsection{Markov Decision Processes}

A Markov decision process (MDP) $\mathcal{M}=\langle Q, \mathrm{~A}, \delta\rangle$ consists of a finite set $Q$ of states, a finite set $\mathrm{A}$ of actions, and a probabilistic transition function $\delta$ : $Q \times \mathrm{A} \rightarrow \mathcal{D}(Q)$. A state $q$ is absorbing if $\delta(q, a)$ is the Dirac distribution on $q$ for all actions $a \in \mathrm{A}$.

We describe the behavior of an MDP as a one-player stochastic game played for infinitely many rounds. Given an initial distribution $\mu_{0} \in \mathcal{D}(Q)$, the game starts in the first round in state $q$ with probability $\mu_{0}(q)$. In each round, the player chooses an action $a \in \mathrm{A}$, and if the game is in state $q$, the next round starts in the successor state $q^{\prime}$ with probability $\delta(q, a)\left(q^{\prime}\right)$.

Given $q \in Q$ and $a \in \mathrm{A}$, denote by $\operatorname{post}(q, a)$ the set $\operatorname{Supp}(\delta(q, a))$, and given $T \subseteq Q$ let $\operatorname{Pre}(T)=\{q \in Q \mid \exists a \in \mathrm{A}: \operatorname{post}(q, a) \subseteq T\}$ be the set of states from which the player has an action to ensure that the successor state is in $T$. For $k>0$, let $\operatorname{Pre}^{k}(T)=\operatorname{Pre}\left(\operatorname{Pre}^{k-1}(T)\right)$ with $\operatorname{Pre}^{0}(T)=T$.

A path in $\mathcal{M}$ is an infinite sequence $\pi=q_{0} a_{0} q_{1} a_{1} \ldots$ such that $q_{i+1} \in$ $\operatorname{post}\left(q_{i}, a_{i}\right)$ for all $i \geq 0$. A finite prefix $\rho=q_{0} a_{0} q_{1} a_{1} \ldots q_{n}$ of a path (or simply a finite path) has length $|\rho|=n$ and last state Last $(\rho)=q_{n}$. We denote by $\operatorname{Play}(\mathcal{M})$ and $\operatorname{Pref}(\mathcal{M})$ the set of all paths and finite paths in $\mathcal{M}$ respectively.

For the decision problems considered in this paper, only the support of the probability distributions in the transition function is relevant (i.e., the exact value of the positive probabilities does not matter); therefore, we can encode an MDP as an A-labelled transition system $(Q, R)$ with $R \subseteq Q \times \mathrm{A} \times Q$ such that $\left(q, a, q^{\prime}\right) \in R$ is a transition if $q^{\prime} \in \operatorname{post}(q, a)$.

Strategies. A randomized strategy for $\mathcal{M}$ (or simply a strategy) is a function $\alpha: \operatorname{Pref}(\mathcal{M}) \rightarrow \mathcal{D}(\mathrm{A})$ that, given a finite path $\rho$, returns a probability distribution $\alpha(\rho)$ over the action set, used to select a successor state $q^{\prime}$ of $\rho$ with probability $\sum_{a \in \mathrm{A}} \alpha(\rho)(a) \cdot \delta(q, a)\left(q^{\prime}\right)$ where $q=\operatorname{Last}(\rho)$.

A strategy $\alpha$ is pure if for all $\rho \in \operatorname{Pref}(\mathcal{M})$, there exists an action $a \in$ A such that $\alpha(\rho)(a)=1$; and memoryless if $\alpha(\rho)=\alpha\left(\rho^{\prime}\right)$ for all $\rho, \rho^{\prime}$ such that Last $(\rho)=$ Last $\left(\rho^{\prime}\right)$. We view pure strategies as functions $\alpha$ : $\operatorname{Pref}(\mathcal{M}) \rightarrow \mathrm{A}$, and memoryless strategies as functions $\alpha: Q \rightarrow \mathcal{D}(\mathrm{A})$, Finally, a strategy $\alpha$ uses finite-memory if it can be represented by a finite-state transducer $T=\left\langle\right.$ Mem, $\left.m_{0}, \alpha_{u}, \alpha_{n}\right\rangle$ where Mem is a finite set of modes (the memory of the strategy), $m_{0} \in$ Mem is the initial mode, $\alpha_{u}:$ Mem $\times \mathrm{A} \times Q \rightarrow$ Mem is an update function, that given the current memory, last action and state updates the memory, and $\alpha_{n}:$ Mem $\times Q \rightarrow \mathcal{D}(\mathrm{A})$ is a next-move function that selects the probability distribution $\alpha_{n}(m, q)$ over actions when the current mode is $m$ and the current state of $\mathcal{M}$ is $q$. For pure strategies, we assume that $\alpha_{n}:$ Mem $\times Q \rightarrow \mathrm{A}$. The memory size of the strategy 
is the number $\mid$ Mem $\mid$ of modes. For a finite-memory strategy $\alpha$, let $\mathcal{M}(\alpha)$ be the Markov chain obtained as the product of $\mathcal{M}$ with the transducer defining $\alpha$.

\subsection{State Semantics}

In the traditional state semantics, given an initial distribution $\mu_{0} \in \mathcal{D}(Q)$ and a strategy $\alpha$ in an MDP $\mathcal{M}$, a path-outcome is a path $\pi=q_{0} a_{0} q_{1} a_{1} \ldots$ in $\mathcal{M}$ such that $q_{0} \in \operatorname{Supp}\left(\mu_{0}\right)$ and $a_{i} \in \operatorname{Supp}\left(\alpha\left(q_{0} a_{0} \ldots a_{i-1} q_{i}\right)\right)$ for all $i \geq 0$. The probability of a finite prefix $\rho=q_{0} a_{0} q_{1} a_{1} \ldots q_{n}$ of $\pi$ is $\mu_{0}\left(q_{0}\right) \cdot \prod_{j=0}^{n-1} \alpha\left(q_{0} a_{0} \ldots q_{j}\right)\left(a_{j}\right)$. $\delta\left(q_{j}, a_{j}\right)\left(q_{j+1}\right)$. We denote by Outcomes $\left(\mu_{0}, \alpha\right)$ the set of all path-outcomes from $\mu_{0}$ under strategy $\alpha$. An event $\Omega \subseteq \operatorname{Play}(\mathcal{M})$ is a measurable set of paths, and given an initial distribution $\mu_{0}$ and a strategy $\alpha$, the probabilities $\operatorname{Pr}^{\alpha}(\Omega)$ of events $\Omega$ are uniquely defined [19]. In particular, given a set $T \subseteq Q$ of target states, and $k \in \mathbb{N}$, we denote by $\square T=\left\{q_{0} a_{0} q_{1} \cdots \in \operatorname{Play}(\mathcal{M}) \mid \forall i: q_{i} \in T\right\}$ the safety event of always staying in $T$, by $\diamond T=\left\{q_{0} a_{0} q_{1} \cdots \in \operatorname{Play}(\mathcal{M}) \mid \exists i: q_{i} \in T\right\}$ the event of reaching $T$, and by $\diamond^{k} T=\left\{q_{0} a_{0} q_{1} \cdots \in \operatorname{Play}(\mathcal{M}) \mid q_{k} \in T\right\}$ the event of reaching $T$ after exactly $k$ steps. Hence, $\operatorname{Pr}^{\alpha}(\diamond T)$ is the probability to reach $T$ under strategy $\alpha$.

We consider the following classical winning modes. Given an initial distribution $\mu_{0}$ and an event $\Omega$, we say that $\mathcal{M}$ is:

- sure winning if there exists a strategy $\alpha$ such that Outcomes $\left(\mu_{0}, \alpha\right) \subseteq \Omega$;

- almost-sure winning if there exists a strategy $\alpha$ such that $\operatorname{Pr}^{\alpha}(\Omega)=1$;

- limit-sure winning if $\sup _{\alpha} \operatorname{Pr}^{\alpha}(\Omega)=1$.

It is known for safety objectives $\square T$ in MDPs that the three winning modes coincide, and for reachability objectives $\diamond T$ that an MDP is almost-sure winning if and only if it is limit-sure winning. For both objectives, the set of initial distributions for which an MDP is sure (resp., almost-sure or limit-sure) winning can be computed in polynomial time [8].

\subsection{Distribution Semantics}

In contrast to the state semantics, we consider the outcome of an MDP $\mathcal{M}$ under a fixed strategy as a sequence of probability distributions over states defined as follows [18. Given an initial distribution $\mu_{0} \in \mathcal{D}(Q)$ and a strategy $\alpha$ in $\mathcal{M}$, the symbolic outcome of $\mathcal{M}$ from $\mu_{0}$ is the sequence $\left(\mathcal{M}_{n}^{\alpha}\right)_{n \in \mathbb{N}}$ of probability distributions defined by $\mathcal{M}_{k}^{\alpha}(q)=\operatorname{Pr}^{\alpha}\left(\diamond^{k}\{q\}\right)$ for all $k \geq 0$ and $q \in Q$. Hence, $\mathcal{M}_{k}^{\alpha}$ is the probability distribution over states after $k$ steps under strategy $\alpha$. Note that $\mathcal{M}_{0}^{\alpha}=\mu_{0}$.

Informally, synchronizing objectives require that the probability of some state (or some group of states) tends to 1 in the sequence $\left(\mathcal{M}_{n}^{\alpha}\right)_{n \in \mathbb{N}}$. Given a set $T \subseteq Q$, consider the functions $\operatorname{sum}_{T}: \mathcal{D}(Q) \rightarrow[0,1]$ and $\max _{T}: \mathcal{D}(Q) \rightarrow[0,1]$ that compute $\operatorname{sum}_{T}(X)=\sum_{q \in T} X(q)$ and $\max _{T}(X)=\max _{q \in T} X(q)$. For $f \in\left\{\operatorname{sum}_{T}, \max _{T}\right\}$ and $p \in[0,1]$, we say that a probability distribution $X$ 
Table 2. Computational complexity of the membership problem, and memory requirement for the strategies (for always synchronizing, the three modes coincide)

\begin{tabular}{|l|c|c|c|c|}
\hline & \multicolumn{2}{|c|}{ Always } & \multicolumn{2}{c|}{ Eventually } \\
\cline { 4 - 5 } & Complexity & Memory requirement & Complexity & Memory requirement \\
\hline \multirow{2}{*}{ Sure } & \multirow{3}{*}{ PTIME-C } & memoryless & PSPACE-C & exponential \\
\cline { 1 - 3 } \cline { 4 - 5 } Almost-sure & & PSPACE-C & infinite \\
\cline { 4 - 5 } & & & PSPACE-C & unbounded \\
\hline
\end{tabular}

is $p$-synchronized according to $f$ if $f(X) \geq p$, and that a sequence $\bar{X}=X_{0} X_{1} \ldots$ of probability distributions is:

(a) always p-synchronizing if $X_{i}$ is $p$-synchronized for all $i \geq 0$;

(b) event (or eventually) p-synchronizing if $X_{i}$ is $p$-synchronized for some $i \geq 0$.

For $p=1$, we view these definitions as the qualitative analogous for sequences of distributions of the traditional safety and reachability conditions for sequences of states 9]. Now, we define the following winning modes. Given an initial distribution $\mu_{0}$ and a function $f \in\left\{s u m_{T}, \max _{T}\right\}$, we say that for the objective of \{always, eventually $\}$ synchronizing from $\mu_{0}, \mathcal{M}$ is:

- sure winning if there exists a strategy $\alpha$ such that the symbolic outcome of $\alpha$ from $\mu_{0}$ is \{always, eventually 1 -synchronizing according to $f$;

- almost-sure winning if there exists a strategy $\alpha$ such that for all $\epsilon>0$ the symbolic outcome of $\alpha$ from $\mu_{0}$ is \{always, eventually $\}(1-\epsilon)$-synchronizing according to $f$;

- limit-sure winning if for all $\epsilon>0$, there exists a strategy $\alpha$ such that the symbolic outcome of $\alpha$ from $\mu_{0}$ is \{always, eventually $(1-\epsilon)$-synchronizing according to $f$;

We often use $X(T)$ instead of $\operatorname{sum}_{T}(X)$, as in Table 1 where the definitions of the various winning modes and synchronizing objectives for $f=s_{\mathrm{s}} \mathrm{m}_{T}$ are summarized. In Section 2.4 we present an example to illustrate the definitions.

\subsection{Decision Problems}

For $f \in\left\{\operatorname{sum}_{T}, \max _{T}\right\}$ and $\lambda \in\{$ always, event $\}$, the winning region $\langle\langle 1\rangle\rangle_{\text {sure }}^{\lambda}(f)$ is the set of initial distributions such that $\mathcal{M}$ is sure winning for $\lambda$-synchronizing (we assume that $\mathcal{M}$ is clear from the context). We define analogously the winning regions $\langle\langle 1\rangle\rangle_{\text {almost }}^{\lambda}(f)$ and $\langle\langle 1\rangle\rangle_{\text {limit }}^{\lambda}(f)$. For a singleton $T=\{q\}$ we have sum $_{T}=$ $\max _{T}$, and we simply write $\langle\langle 1\rangle\rangle_{\mu}^{\lambda}(q)$ (where $\mu \in\{$ sure, almost, limit $\}$ ). We are interested in the algorithmic complexity of the membership problem, which is to decide, given a probability distribution $\mu_{0}$, whether $\mu_{0} \in\langle\langle 1\rangle\rangle_{\mu}^{\lambda}(f)$. As we show below, it is easy to establish the complexity of the membership problems for always synchronizing, while it is more tricky for eventually synchronizing. The complexity results are summarized in Table 2 . 


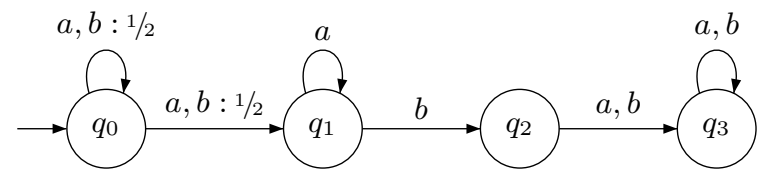

Fig. 1. An MDP $\mathcal{M}$ such that $\langle\langle 1\rangle\rangle_{\text {sure }}^{\text {event }}\left(q_{1}\right) \neq\langle\langle 1\rangle\rangle_{\text {almost }}^{\text {event }}\left(q_{1}\right)$ and $\langle\langle 1\rangle\rangle_{\text {almost }}^{\text {event }}\left(q_{2}\right) \neq$ $\langle\langle 1\rangle\rangle_{\text {limit }}^{\text {event }}\left(q_{2}\right)$

Always Synchronizing. We first remark that for always synchronizing, the three winning modes coincide.

Lemma 1. Let $T$ be a set of states. For all functions $f \in\left\{\max _{T}\right.$, sum $\left._{T}\right\}$, we have $\langle\langle 1\rangle\rangle_{\text {sure }}^{\text {always }}(f)=\langle\langle 1\rangle\rangle_{\text {almost }}^{\text {always }}(f)=\langle\langle 1\rangle\rangle_{\text {limit }}^{\text {always }}(f)$.

It follows from the proof of Lemma 1 that the winning region for always synchronizing according to sum $_{T}$ coincides with the set of winning initial distributions for the safety objective $\square T$ in the traditional state semantics, which can be computed in polynomial time [7. Moreover, always synchronizing according to $\max _{T}$ is equivalent to the existence of an infinite path staying in $T$ in the transition system $\langle Q, R\rangle$ of the MDP restricted to transitions $\left(q, a, q^{\prime}\right) \in R$ such that $\delta(q, a)\left(q^{\prime}\right)=1$, which can also be decided in polynomial time. In both cases, pure memoryless strategies are sufficient.

Theorem 1. The membership problem for always synchronizing can be solved in polynomial time, and pure memoryless strategies are sufficient.

Eventually Synchronizing. For all functions $f \in\left\{\max _{T}, \operatorname{sum}_{T}\right\}$, the following inclusions hold: $\langle\langle 1\rangle\rangle_{\text {sure }}^{\text {event }}(f) \subseteq\langle\langle 1\rangle\rangle_{\text {almost }}^{\text {event }}(f) \subseteq\langle\langle 1\rangle\rangle_{\text {limit }}^{\text {event }}(f)$ and we show that the inclusions are strict in general. Consider the MDP in Fig. 1] with initial state $q_{0}$ and target $T=\left\{q_{1}\right\}$. For all strategies, the probability in $q_{0}$ is always positive, implying that the MDP is not sure-winning in $\left\{q_{1}\right\}$. However, the MDP is almost-sure winning in $\left\{q_{1}\right\}$ using a strategy that always plays $a$. Now, consider target $T=\left\{q_{2}\right\}$. For all $\epsilon>0$, we can have probability at least $1-\epsilon$ in $q_{2}$ by playing $a$ long enough, and then $b$. For a fixed strategy, this probability never tends to 1 since if the probability $p>0$ in $q_{2}$ is positive at a certain step, then it remains bounded by $1-p<1$ for all next steps. Therefore, the MDP is not almost-sure winning in $\left\{q_{2}\right\}$, but it is limit-sure winning.

Lemma 2. There exists an $M D P \mathcal{M}$ and states $q_{1}, q_{2}$ such that:

(i) $\langle\langle 1\rangle\rangle_{\text {sure }}^{\text {event }}\left(q_{1}\right) \subsetneq\langle\langle 1\rangle\rangle_{\text {almost }}^{\text {event }}\left(q_{1}\right)$, and

(ii) $\langle\langle 1\rangle\rangle_{\text {almost }}^{\text {event }}\left(q_{2}\right) \subsetneq\langle\langle 1\rangle\rangle_{\text {limit }}^{\text {event }}\left(q_{2}\right)$.

The rest of this paper is devoted to the solution of the membership problem for eventually synchronizing. We make some preliminary remarks to show that it is sufficient to solve the membership problem according to $f=s_{T} m_{T}$ and for MDPs with a single initial state. Our results will also show that pure strategies are sufficient in all modes. 
Remark. For eventually synchronizing and each winning mode, we show that the membership problem with function $\max _{T}$ is polynomial-time equivalent to the membership problem with function sum $_{T^{\prime}}$ with a singleton $T^{\prime}$. First, for $\mu \in\{$ sure, almost, limit $\}$, we have $\langle\langle 1\rangle\rangle_{\mu}^{\text {event }}\left(\max _{T}\right)=\bigcup_{q \in T}\langle\langle 1\rangle\rangle_{\mu}^{\text {event }}(q)$, showing that the membership problems for $\max$ are polynomial-time reducible to the corresponding membership problem for sum $T_{T^{\prime}}$ with singleton $T^{\prime}$. The reverse reduction is as follows. Given an $\mathrm{MDP} \mathcal{M}^{\prime}$, a singleton $T^{\prime}=\{q\}$ and an initial distribution $\mu_{0}^{\prime}$, we can construct an MDP $\mathcal{M}$ and initial distribution $\mu_{0}$ such that $\mu_{0}^{\prime} \in\langle\langle 1\rangle\rangle_{\mu}^{\text {event }}(q)$ iff $\mu_{0} \in\langle\langle 1\rangle\rangle_{\mu}^{\text {event }}\left(\max _{T}\right)$ where $T=Q$ is the state space of $\mathcal{M}$. The idea is to construct $\mathcal{M}$ and $\mu_{0}$ as a copy of $\mathcal{M}^{\prime}$ and $\mu_{0}^{\prime}$ where all states except $q$ are duplicated, and the initial and transition probabilities are evenly distributed between the copies. Therefore if the probability tends to 1 in some state, it has to be in $q$.

Remark. To solve the membership problems for eventually synchronizing with function $\operatorname{sum}_{T}$, it is sufficient to provide an algorithm that decides membership of Dirac distributions (i.e., assuming MDPs have a single initial state), since to solve the problem for an MDP $\mathcal{M}$ with initial distribution $\mu_{0}$, we can equivalently solve it for a copy of $\mathcal{M}$ with a new initial state $q_{0}$ from which the successor distribution on all actions is $\mu_{0}$. Therefore, it is sufficient to consider initial Dirac distributions $\mu_{0}$.

\section{One-Letter Alternating Automata}

In this section, we consider one-letter alternating automata (1L-AFA) as they have a structure of alternating graph analogous to MDP (i.e., when ignoring the probabilities). We review classical decision problems for 1L-AFA, and establish the complexity of a new problem, the universal finiteness problem which is to decide if from every initial state the language of a given 1L-AFA is finite. These results of independent interest are useful to establish the PSPACE lower bounds for eventually synchronizing in MDPs.

One-Letter Alternating Automata. Let $\mathrm{B}^{+}(Q)$ be the set of positive Boolean formulas over $Q$, i.e. Boolean formulas built from elements in $Q$ using $\wedge$ and $\vee$. A set $S \subseteq Q$ satisfies a formula $\varphi \in \mathrm{B}^{+}(Q)$ (denoted $S \models \varphi$ ) if $\varphi$ is satisfied when replacing in $\varphi$ the elements in $S$ by true, and the elements in $Q \backslash S$ by false.

A one-letter alternating finite automaton is a tuple $\mathcal{A}=\left\langle Q, \delta_{\mathcal{A}}, \mathcal{F}\right\rangle$ where $Q$ is a finite set of states, $\delta_{\mathcal{A}}: Q \rightarrow \mathrm{B}^{+}(Q)$ is the transition function, and $\mathcal{F} \subseteq Q$ is the set of accepting states. We assume that the formulas in transition function are in disjunctive normal form. Note that the alphabet of the automaton is omitted, as it has a single letter. In the language of a 1L-AFA, only the length of words is relevant. For all $n \geq 0$, define the set $A c c_{\mathcal{A}}(n, \mathcal{F}) \subseteq Q$ of states from which the word of length $n$ is accepted by $\mathcal{A}$ as follows: 
$-\operatorname{Acc}_{\mathcal{A}}(0, \mathcal{F})=\mathcal{F}$

- $A c c_{\mathcal{A}}(n, \mathcal{F})=\left\{q \in Q\left|A c c_{\mathcal{A}}(n-1, \mathcal{F})\right|=\delta(q)\right\}$ for all $n>0$.

The set $\mathcal{L}\left(\mathcal{A}_{q}\right)=\left\{n \in \mathbb{N} \mid q \in A c c_{\mathcal{A}}(n, \mathcal{F})\right\}$ is the language accepted by $\mathcal{A}$ from initial state $q$.

For fixed $n$, we view $A c c_{\mathcal{A}}(n, \cdot)$ as an operator on $2^{Q}$ that, given a set $\mathcal{F} \subseteq Q$ computes the set $A c c_{\mathcal{A}}(n, \mathcal{F})$. Note that $A c c_{\mathcal{A}}(n, \mathcal{F})=A c c_{\mathcal{A}}\left(1, A c c_{\mathcal{A}}(n-1, \mathcal{F})\right)$ for all $n \geq 1$. Denote by $\operatorname{Pre}_{\mathcal{A}}(\cdot)$ the operator $A c c_{\mathcal{A}}(1, \cdot)$. Then for all $n \geq 0$ the operator $A c c_{\mathcal{A}}(n, \cdot)$ coincides with $\operatorname{Pre}_{\mathcal{A}}^{n}(\cdot)$, the $n$-th iterate of $\operatorname{Pre}_{\mathcal{A}}(\cdot)$.

Decision Problems. We present the classical emptiness and finiteness problems for alternating automata, and we introduce a variant of the finiteness problem that will be useful for solving synchronizing problems for MDPs.

- The emptiness problem for 1L-AFA is to decide, given a 1L-AFA $\mathcal{A}$ and an initial state $q$, whether $\mathcal{L}\left(\mathcal{A}_{q}\right)=\emptyset$. The emptiness problem can be solved by checking whether $q \in \operatorname{Pre}_{\mathcal{A}}^{n}(\mathcal{F})$ for some $n \geq 0$. It is known that the emptiness problem is PSPACE-complete, even for transition functions in disjunctive normal form [1617].

- The finiteness problem is to decide, given a 1L-AFA $\mathcal{A}$ and an initial state $q$, whether $\mathcal{L}\left(\mathcal{A}_{q}\right)$ is finite. The sequence $\operatorname{Pre}_{\mathcal{A}}^{n}(\mathcal{F})$ is ultimately periodic, and for all $n \geq 0$, there exists $n_{0} \leq 2^{|Q|}$ such that $\operatorname{Pre}_{\mathcal{A}}^{n_{0}}(\mathcal{F})=\operatorname{Pre}_{\mathcal{A}}^{n}(\mathcal{F})$. Therefore, the finiteness problem can be solved in (N)PSPACE by guessing $n, k \leq 2^{|Q|}$ such that $\operatorname{Pre}_{\mathcal{A}}^{n+k}(\mathcal{F})=\operatorname{Pre}_{\mathcal{A}}^{n}(\mathcal{F})$ and $q \in \operatorname{Pre}_{\mathcal{A}}^{n}(\mathcal{F})$. The finiteness problem is PSPACE-complete by a simple reduction from the emptiness problem: from an instance $(\mathcal{A}, q)$ of the emptiness problem, construct $\left(\mathcal{A}^{\prime}, q^{\prime}\right)$ where $q^{\prime}=q$ and $\mathcal{A}^{\prime}=\left\langle Q, \delta^{\prime}, \mathcal{F}\right\rangle$ is a copy of $\mathcal{A}=\langle Q, \delta, \mathcal{F}\rangle$ with a self-loop on $q$ (formally, $\delta^{\prime}(q)=q \vee \delta(q)$ and $\delta^{\prime}(r)=\delta(r)$ for all $\left.r \in Q \backslash\{q\}\right)$. It is easy to see that $\mathcal{L}\left(\mathcal{A}_{q}\right)=\emptyset$ iff $\mathcal{L}\left(\mathcal{A}_{q^{\prime}}^{\prime}\right)$ is finite.

- The universal finiteness problem is to decide, given a $1 \mathrm{~L}-\mathrm{AFA} \mathcal{A}$, whether $\mathcal{L}\left(\mathcal{A}_{q}\right)$ is finite for all states $q$. This problem can be solved by checking whether $\operatorname{Pre}_{\mathcal{A}}^{n}(\mathcal{F})=\emptyset$ for some $n \leq 2^{|Q|}$, and thus it is in PSPACE. Note that if $\operatorname{Pre}_{\mathcal{A}}^{n}(\mathcal{F})=\emptyset$, then $\operatorname{Pre}_{\mathcal{A}}^{m}(\mathcal{F})=\emptyset$ for all $m \geq n$.

Given the PSPACE-hardness proofs of the emptiness and finiteness problems, it is not easy to see that the universal finiteness problem is PSPACE-hard.

Lemma 3. The universal finiteness problem for $1 L-A F A$ is PSPACE-hard.

Relation with MDPs. The underlying structure of a Markov decision process $\mathcal{M}=\langle Q, \mathrm{~A}, \delta\rangle$ is an alternating graph, where the successor $q^{\prime}$ of a state $q$ is obtained by an existential choice of an action $a$ and a universal choice of a state $q^{\prime} \in \operatorname{Supp}(\delta(q, a))$. Therefore, it is natural that some questions related to MDPs have a corresponding formulation in terms of alternating automata. We show that such connections exist between synchronizing problems for MDPs and language-theoretic questions for alternating automata, such as emptiness and universal finiteness. Given a $1 \mathrm{~L}-\mathrm{AFA} \mathcal{A}=\left\langle Q, \delta_{\mathcal{A}}, \mathcal{F}\right\rangle$, assume without loss 
of generality that the transition function $\delta_{\mathcal{A}}$ is such that $\delta_{\mathcal{A}}(q)=c_{1} \vee \cdots \vee c_{m}$ has the same number $m$ of conjunctive clauses for all $q \in Q$. From $\mathcal{A}$, construct the $\operatorname{MDP} \mathcal{M}_{\mathcal{A}}=\left\langle Q, \mathrm{~A}, \delta_{\mathcal{M}}\right\rangle$ where $\mathrm{A}=\left\{a_{1}, \ldots, a_{m}\right\}$ and $\delta_{\mathcal{M}}\left(q, a_{k}\right)$ is the uniform distribution over the states occurring in the $k$-th clause $c_{k}$ in $\delta_{\mathcal{A}}(q)$, for all $q \in Q$ and $a_{k} \in \mathrm{A}$. Then, we have $\operatorname{Acc}_{\mathcal{A}}(n, \mathcal{F})=\operatorname{Pre}_{\mathcal{M}}^{n}(\mathcal{F})$ for all $n \geq 0$. Similarly, from an MDP $\mathcal{M}$ and a set $T$ of states, we can construct a $1 \mathrm{~L}-\mathrm{AFA}$ $\mathcal{A}=\left\langle Q, \delta_{\mathcal{A}}, \mathcal{F}\right\rangle$ with $\mathcal{F}=T$ such that $A c c_{\mathcal{A}}(n, \mathcal{F})=\operatorname{Pre}_{\mathcal{M}}^{n}(T)$ for all $n \geq 0$ (let $\delta_{\mathcal{A}}(q)=\bigvee_{a \in \mathrm{A}} \bigwedge_{q^{\prime} \in \operatorname{post}(q, a)} q^{\prime}$ for all $\left.q \in Q\right)$.

Several decision problems for 1L-AFA can be solved by computing the sequence $A c c_{\mathcal{A}}(n, \mathcal{F})$, and we show that some synchronizing problems for MDPs require the computation of the sequence $\operatorname{Pre}_{\mathcal{M}}^{n}(\mathcal{F})$. Therefore, the above relation between 1L-AFA and MDPs establishes bridges that we use in Section 4 to transfer complexity results from 1L-AFA to MDPs.

\section{Eventually Synchronization}

In this section, we show the PSPACE-completeness of the membership problem for eventually synchronizing objectives and the three winning modes. By the remarks at the end of Section 2, we consider the membership problem with function sum and Dirac initial distributions (i.e., single initial state).

\subsection{Sure Eventually Synchronization}

Given a target set $T$, the membership problem for sure-winning eventually synchronizing objective in $T$ can be solved by computing the sequence $\operatorname{Pre}^{n}(T)$ of iterated predecessor. A state $q_{0}$ is sure-winning for eventually synchronizing in $T$ if $q_{0} \in \operatorname{Pre}^{n}(T)$ for some $n \geq 0$.

Lemma 4. Let $\mathcal{M}$ be an $M D P$ and $T$ be a target set. For all states $q_{0}$, we have $q_{0} \in\langle\langle 1\rangle\rangle_{\text {sure }}^{\text {event }}\left(\right.$ sum $\left._{T}\right)$ if and only if there exists $n \geq 0$ such that $q_{0} \in \operatorname{Pre}_{\mathcal{M}}^{n}(T)$.

By Lemma 4, the membership problem for sure eventually synchronizing is equivalent to the emptiness problem of 1L-AFA, and thus PSPACE-complete (even when $T$ is a singleton). Moreover if $q_{0} \in \operatorname{Pre}_{\mathcal{M}}^{n}(T)$, a finite-memory strategy with $n$ modes that at mode $i$ in a state $q$ plays an action $a$ such that $\operatorname{post}(q, a) \subseteq$ $\operatorname{Pre}^{i-1}(T)$ is sure winning for eventually synchronizing. There exists a family of $\operatorname{MDPs} \mathcal{M}_{n}(n \in \mathbb{N})$ that are sure winning for eventually synchronization, and where the sure winning strategies require exponential memory [12. Essentially, the structure of $\mathcal{M}_{n}$ is an initial uniform probabilistic transition to $n$ components $H_{1}, \ldots, H_{n}$ where $H_{i}$ is a cycle of length $p_{i}$ the $i$-th prime number, and sure eventually synchronization requires memory size $p_{n}^{\#}=\prod_{i=1}^{n} p_{i}$. The following theorem summarizes the results for sure eventually synchronizing.

Theorem 2. For sure eventually synchronizing in MDPs:

1. (Complexity). The membership problem is PSPACE-complete.

2. (Memory). Exponential memory is necessary and sufficient for both pure and randomized strategies, and pure strategies are sufficient. 


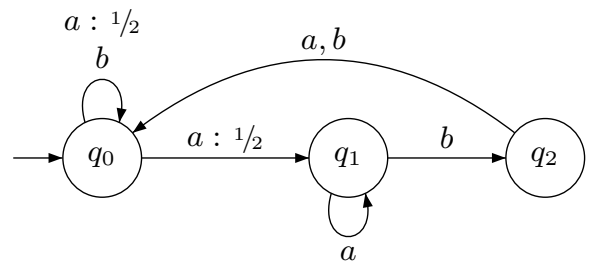

Fig. 2. An MDP where infinite memory is necessary for almost-sure eventually synchronizing strategies

\subsection{Almost-Sure Eventually Synchronization}

We show an example where infinite memory is necessary to win for almostsure eventually synchronizing. Consider the MDP in Fig. 2 with initial state $q_{0}$. We construct a strategy that is almost-sure eventually synchronizing in $\left\{q_{2}\right\}$, showing that $q_{0} \in\langle\langle 1\rangle\rangle_{\text {almost }}^{\text {event }}\left(q_{2}\right)$. First, observe that for all $\epsilon>0$ we can have probability at least $1-\epsilon$ in $q_{2}$ after finitely many steps: playing $n$ times $a$ and then $b$ leads to probability $1-\frac{1}{2^{n}}$ in $q_{2}$. Thus the MDP is limit-sure eventually synchronizing in $q_{2}$. Moreover the remaining probability mass is in $q_{0}$. It turns out that from any (initial) distribution with support $\left\{q_{0}, q_{2}\right\}$, the MDP is again limitsure eventually synchronizing in $q_{2}$ (and with support in $\left\{q_{0}, q_{2}\right\}$ ). Therefore we can take a smaller value of $\epsilon$ and play a strategy to have probability at least $1-\epsilon$ in $q_{2}$, and repeat this for $\epsilon \rightarrow 0$. This strategy ensures almost-sure eventually synchronizing in $q_{2}$. The next result shows that infinite memory is necessary for almost-sure winning in this example.

Lemma 5. There exists an almost-sure eventually synchronizing MDP for which all almost-sure eventually synchronizing strategies require infinite memory.

It turns out that in general, almost-sure eventually synchronizing strategies can be constructed from a family of limit-sure eventually synchronizing strategies if we can also ensure that the probability mass remains in the winning region (as in the MDP in Fig. (2). We present a characterization of the winning region for almost-sure winning based on an extension of the limit-sure eventually synchronizing objective with exact support. This objective requires to ensure probability arbitrarily close to 1 in the target set $T$, and moreover that after the same number of steps the support of the probability distribution is contained in a given set $U$. Formally, given an MDP $\mathcal{M}$, let $\langle\langle 1\rangle\rangle_{\text {limit }}^{\text {event }}\left(\operatorname{sum}_{T}, U\right)$ for $T \subseteq U$ be the set of all initial distributions such that for all $\epsilon>0$ there exists a strategy $\alpha$ and $n \in \mathbb{N}$ such that $\mathcal{M}_{n}^{\alpha}(T) \geq 1-\epsilon$ and $\mathcal{M}_{n}^{\alpha}(U)=1$. We say that $\alpha$ is limit-sure eventually synchronizing in $T$ with support in $U$.

We will present an algorithmic solution to limit-sure eventually synchronizing objectives with exact support in Section 4.3. Our characterization of the winning region for almost-sure winning is as follows. There must exist a support $U$ such that $(i)$ the MDP is sure winning for eventually synchronizing in target $U$, 
and (ii) from distributions with support in $U$, it is possible to get probability arbitrarily close to 1 in $T$, and support back in $U$. In the example of Fig. 2 with $T=\left\{q_{2}\right\}$, we can take $U=\left\{q_{0}, q_{2}\right\}$.

Lemma 6. Let $\mathcal{M}$ be an $M D P$ and $T$ be a target set. For all states $q_{0}$, we have $q_{0} \in\langle\langle 1\rangle\rangle_{\text {almost }}^{\text {event }}\left(\right.$ sum $\left._{T}\right)$ if and only if there exists a set $U$ such that:

- $q_{0} \in\langle\langle 1\rangle\rangle_{\text {sure }}^{\text {event }}\left(\right.$ sum $\left._{U}\right)$, and

$-d_{U} \in\langle\langle 1\rangle\rangle_{\text {limit }}^{\text {event }}\left(\right.$ sum $\left._{T}, U\right)$ where $d_{U}$ is the uniform distribution over $U$.

As we show in Section 4.3 that the membership problem for limit-sure eventually synchronizing with exact support can be solved in PSPACE, it follows from the characterization in Lemma 6 that the membership problem for almostsure eventually synchronizing is in PSPACE, using the following (N)PSPACE algorithm: guess the set $U$, and check that $q_{0} \in\langle\langle 1\rangle\rangle_{\text {sure }}^{\text {event }}\left(\right.$ sum $\left._{U}\right)$, and that $d_{U} \in\langle\langle 1\rangle\rangle_{\text {limit }}^{\text {event }}\left(\right.$ sum $\left._{T}, U\right)$ where $d_{U}$ is the uniform distribution over $U$ (both can be done in PSPACE by Theorem 2 and Theorem 4). We present a matching lower bound using a reduction from the membership problem for sure eventually synchronization [12, which is PSPACE-complete by Theorem 2,

Lemma 7. The membership problem for $\langle\langle 1\rangle\rangle_{\text {almont }}^{\text {event }}\left(\right.$ sum $\left._{T}\right)$ is PSPACE-hard even if $T$ is a singleton.

The results of this section are summarized as follows.

Theorem 3. For almost-sure eventually synchronizing in MDPs:

1. (Complexity). The membership problem is PSPACE-complete.

2. (Memory). Infinite memory is necessary in general for both pure and randomized strategies, and pure strategies are sufficient.

\subsection{Limit-Sure Eventually Synchronization}

In this section, we present the algorithmic solution for limit-sure eventually synchronizing with exact support. Note that the limit-sure eventually synchronizing objective is a special case where the support is the state space of the MDP. Consider the MDP in Fig. 1 which is limit-sure eventually synchronizing in $\left\{q_{2}\right\}$, as shown in Lemma 2. For $i=0,1, \ldots$, the sequence $\operatorname{Pre}^{i}(T)$ of predecessors of $T=\left\{q_{2}\right\}$ is ultimately periodic: $\operatorname{Pre}^{0}(T)=\left\{q_{2}\right\}$, and $\operatorname{Pre}^{i}(T)=\left\{q_{1}\right\}$ for all $i \geq 1$. Given $\epsilon>0$, a strategy to get probability $1-\epsilon$ in $q_{2}$ first accumulates probability mass in the periodic subsequence of predecessors (here $\left\{q_{1}\right\}$ ), and when the probability mass is greater than $1-\epsilon$ in $q_{1}$, the strategy injects the probability mass in $q_{2}$ (through the aperiodic prefix of the sequence of predecessors). This is the typical shape of a limit-sure eventually synchronizing strategy. Note that in this scenario, the MDP is also limit-sure eventually synchronizing in every set $\operatorname{Pre}^{i}(T)$ of the sequence of predecessors. A special case is when it is possible to get probability 1 in the sequence of predecessors after finitely 
many steps. In this case, the probability mass injected in $T$ is 1 and the MDP is even sure-winning. The algorithm for deciding limit-sure eventually synchronization relies on the above characterization, generalized in Lemma 8 to limit-sure eventually synchronizing with exact support, saying that limit-sure eventually synchronizing in $T$ with support in $U$ is equivalent to either limit-sure eventually synchronizing in $\operatorname{Pre}^{k}(T)$ with support in $\operatorname{Pre}^{k}(U)$ (for arbitrary $k$ ), or sure eventually synchronizing in $T$ (and therefore also in $U$ ).

Lemma 8. For all $T \subseteq U$ and all $k \geq 0$, we have $\langle\langle 1\rangle\rangle_{\text {limit }}^{\text {event }}\left(\right.$ sum $\left._{T}, U\right)=$ $\langle\langle 1\rangle\rangle_{\text {sure }}^{\text {event }}\left(\right.$ sum $\left._{T}\right) \cup\langle\langle 1\rangle\rangle_{\text {limit }}^{\text {event }}\left(\right.$ sum $\left._{R}, Z\right)$ where $R=\operatorname{Pre}^{k}(T)$ and $Z=\operatorname{Pre}^{k}(U)$.

Thanks to Lemma 8, since sure-winning is already solved in Section 4.1, it suffices to solve the limit-sure eventually synchronizing problem for target $R=$ $\operatorname{Pre}^{k}(T)$ and support $Z=\operatorname{Pre}^{k}(U)$ with arbitrary $k$, instead of $T$ and $U$. We can choose $k$ such that both $\operatorname{Pre}^{k}(T)$ and $\operatorname{Pre}^{k}(U)$ lie in the periodic part of the sequence of pairs of predecessors $\left(\operatorname{Pre}^{i}(T), \operatorname{Pre}^{i}(U)\right)$. We can assume that $k \leq 3^{|Q|}$ since $\operatorname{Pre}^{i}(T) \subseteq \operatorname{Pre}^{i}(U) \subseteq Q$ for all $i \geq 0$. For such value of $k$ the limit-sure problem is conceptually simpler: once some probability is injected in $R=\operatorname{Pre}^{k}(T)$, it can loop through the sequence of predecessors and visit $R$ infinitely often (every $r$ steps, where $r \leq 3^{|Q|}$ is the period of the sequence of pairs of predecessors). It follows that if a strategy ensures with probability 1 that the set $R$ can be reached by finite paths whose lengths are congruent modulo $r$, then the whole probability mass can indeed synchronously accumulate in $R$ in the limit. Therefore, limit-sure eventually synchronizing in $R$ reduces to standard limit-sure reachability with target set $R$ and the additional requirement that the numbers of steps at which the target set is reached be congruent modulo $r$. In the case of limit-sure eventually synchronizing with support in $Z$, we also need to ensure that no mass of probability leaves the sequence $\operatorname{Pre}^{i}(Z)$. In a state $q \in \operatorname{Pre}^{i}(Z)$, we say that an action $a \in \mathrm{A}$ is $Z$-safe at position $i$ if $\operatorname{post}(q, a) \subseteq$ $\operatorname{Pre}^{i-1}(Z)$. In states $q \notin \operatorname{Pre}^{i}(Z)$ there is no $Z$-safe action at position $i$.

To encode the above requirements, we construct an $\mathrm{MDP} \mathcal{M}_{Z} \times[r]$ that allows only $Z$-safe actions to be played (and then mimics the original MDP), and tracks the position (modulo $r$ ) in the sequence of predecessors, thus simply decrementing the position on each transition since all successors of a state $q \in$ $\operatorname{Pre}^{i}(Z)$ on a safe action are in $\operatorname{Pre}^{i-1}(Z)$. Formally, if $\mathcal{M}=\langle Q, \mathrm{~A}, \delta\rangle$ then $\mathcal{M}_{Z} \times[r]=\left\langle Q^{\prime}, \mathrm{A}, \delta^{\prime}\right\rangle$ where:

- $Q^{\prime}=Q \times\{r-1, \ldots, 1,0\} \cup\{$ sink $\}$; intuitively, we expect that $q \in \operatorname{Pre}^{i}(Z)$ in the reachable states $\langle q, i\rangle$ consisting of a state $q$ of $\mathcal{M}$ and a position $i$ in the predecessor sequence;

- $\delta^{\prime}$ is defined as follows (assuming an arithmetic modulo $r$ on positions) for all $\langle q, i\rangle \in Q^{\prime}$ and $a \in \mathrm{A}$ : if $a$ is a $Z$-safe action in $q$ at position $i$, then $\delta^{\prime}(\langle q, i\rangle, a)\left(\left\langle q^{\prime}, i-1\right\rangle\right)=\delta(q, a)\left(q^{\prime}\right)$, otherwise $\delta^{\prime}(\langle q, i\rangle, a)($ sink $)=1$ (and sink is absorbing).

${ }^{1}$ Since $\operatorname{Pre}^{r}(Z)=Z$ and $\operatorname{Pre}^{r}(R)=R$, we assume a modular arithmetic for exponents

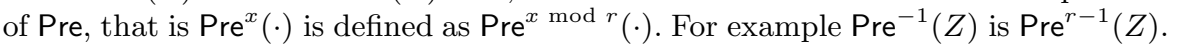


Note that the size of the $\operatorname{MDP} \mathcal{M}_{Z} \times[r]$ is exponential in the size of $\mathcal{M}$ (since $r$ is at most $3^{|Q|}$ ).

Lemma 9. Let $\mathcal{M}$ be an $M D P$ and $R \subseteq Z$ be two sets of states such that $\operatorname{Pre}^{r}(R)=R$ and $\operatorname{Pre}^{r}(Z)=Z$ where $r>0$. Then a state $q_{0}$ is limit-sure eventually synchronizing in $R$ with support in $Z\left(q_{0} \in\langle\langle 1\rangle\rangle_{\text {limit }}^{\text {event }}\left(\right.\right.$ sum $\left.\left._{R}, Z\right)\right)$ if and only if there exists $0 \leq t<r$ such that $\left\langle q_{0}, t\right\rangle$ is limit-sure winning for the reachability objective $\diamond(R \times\{0\})$ in the $M D P \mathcal{M}_{Z} \times[r]$.

Since deciding limit-sure reachability is PTIME-complete, it follows from Lemma 9 that limit-sure synchronization (with exact support) can be decided in EXPTIME. We can show that the problem can be solved in PSPACE by exploiting the special structure of the exponential MDP in Lemma 9.

Lemma 10. The membership problem for limit-sure eventually synchronization with exact support is in PSPACE.

To establish the PSPACE-hardness for limit-sure eventually synchronizing in MDPs, we use a reduction from the universal finiteness problem for 1L-AFAs.

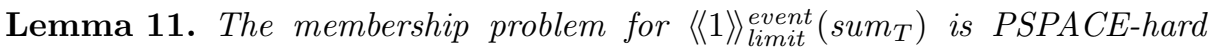
even if $T$ is a singleton.

The example in Fig. 2 can be used to show that the memory needed by a family of strategies to win limit-sure eventually synchronizing objective (in target $T=\left\{q_{2}\right\}$ ) is unbounded.

Theorem 4. For limit-sure eventually synchronizing (with or without exact support) in MDPs:

1. (Complexity). The membership problem is PSPACE-complete.

2. (Memory). Unbounded memory is required for both pure and randomized strategies, and pure strategies are sufficient.

Acknowledgment. We are grateful to Winfried Just and German A. Enciso for helpful discussions on Boolean networks and for the gadget in the proof of Lemma 3.

\section{References}

1. Agrawal, M., Akshay, S., Genest, B., Thiagarajan, P.S.: Approximate verification of the symbolic dynamics of Markov chains. In: LICS, pp. 55-64. IEEE (2012)

2. Aspnes, J., Herlihy, M.: Fast randomized consensus using shared memory. J. Algorithm 11(3), 441-461 (1990)

3. Baier, C., Bertrand, N., Größer, M.: On decision problems for probabilistic Büchi automata. In: Amadio, R.M. (ed.) FOSSACS 2008. LNCS, vol. 4962, pp. 287-301. Springer, Heidelberg (2008) 
4. Baier, C., Bertrand, N., Schnoebelen, P.: On computing fixpoints in well-structured regular model checking, with applications to lossy channel systems. In: Hermann, M., Voronkov, A. (eds.) LPAR 2006. LNCS (LNAI), vol. 4246, pp. 347-361. Springer, Heidelberg (2006)

5. Baldoni, R., Bonnet, F., Milani, A., Raynal, M.: On the solvability of anonymous partial grids exploration by mobile robots. In: Baker, T.P., Bui, A., Tixeuil, S. (eds.) OPODIS 2008. LNCS, vol. 5401, pp. 428-445. Springer, Heidelberg (2008)

6. Chadha, R., Korthikanti, V.A., Viswanathan, M., Agha, G., Kwon, Y.: Model checking MDPs with a unique compact invariant set of distributions. In: Proc. of QEST, pp. 121-130. IEEE Computer Society (2011)

7. Chatterjee, K., Henzinger, T.A.: A survey of stochastic $\omega$-regular games. J. Comput. Syst. Sci. 78(2), 394-413 (2012)

8. de Alfaro, L.: Formal Verification of Probabilistic Systems. PhD thesis, Stanford University (1997)

9. de Alfaro, L., Henzinger, T.A., Kupferman, O.: Concurrent reachability games. Theor. Comput. Sci. 386(3), 188-217 (2007)

10. Doyen, L., Massart, T., Shirmohammadi, M.: Infinite synchronizing words for probabilistic automata. In: Murlak, F., Sankowski, P. (eds.) MFCS 2011. LNCS, vol. 6907, pp. 278-289. Springer, Heidelberg (2011)

11. Doyen, L., Massart, T., Shirmohammadi, M.: Infinite synchronizing words for probabilistic automata (Erratum). CoRR, abs/1206.0995 (2012)

12. Doyen, L., Massart, T., Shirmohammadi, M.: Limit synchronization in Markov decision processes. CoRR, abs/1310.2935 (2013)

13. Fokkink, W., Pang, J.: Variations on Itai-Rodeh leader election for anonymous rings and their analysis in PRISM. Journal of Universal Computer Science 12(8), 981-1006 (2006)

14. Gimbert, H., Oualhadj, Y.: Probabilistic automata on finite words: Decidable and undecidable problems. In: Abramsky, S., Gavoille, C., Kirchner, C., Meyer auf der Heide, F., Spirakis, P.G. (eds.) ICALP 2010, part II. LNCS, vol. 6199, pp. 527-538. Springer, Heidelberg (2010)

15. Henzinger, T.A., Mateescu, M., Wolf, V.: Sliding window abstraction for infinite Markov chains. In: Bouajjani, A., Maler, O. (eds.) CAV 2009. LNCS, vol. 5643, pp. 337-352. Springer, Heidelberg (2009)

16. Holzer, M.: On emptiness and counting for alternating finite automata. In: Developments in Language Theory, pp. 88-97 (1995)

17. Jancar, P., Sawa, Z.: A note on emptiness for alternating finite automata with a one-letter alphabet. Inf. Process. Lett. 104(5), 164-167 (2007)

18. Korthikanti, V.A., Viswanathan, M., Agha, G., Kwon, Y.: Reasoning about MDPs as transformers of probability distributions. In: Proc. of QEST, pp. 199-208. IEEE Computer Society (2010)

19. Vardi, M.Y.: Automatic verification of probabilistic concurrent finite-state programs. In: Proc. of FOCS, pp. 327-338. IEEE Computer Society (1985)

20. Volkov, M.V.: Synchronizing automata and the Černý conjecture. In: Martín-Vide, C., Otto, F., Fernau, H. (eds.) LATA 2008. LNCS, vol. 5196, pp. 11-27. Springer, Heidelberg (2008) 